\title{
SUPERMINIATURE EDDY-CURRENT TRANSDUCERS FOR THICKNESS STUDIES
}

\author{
S. F. Dmitriev ${ }^{1}$, A. V. Ishkov ${ }^{2}$, A. O. Katasonov ${ }^{1}$, \\ V. N. Malikov ${ }^{1 *}$ A. M. Sagalakov ${ }^{1}$ \\ ${ }^{1}$ Altai State University, 61 Lenina St., Barnaul, Russian Federation \\ ${ }^{2}$ Altai State Agrarian University, 98 Krasnoarmeyskiy Ave., Barnaul, Russian Federation \\ *Corresponding author. E-mail: osys@me.com \\ Address for correspondence: Krasnoarmeysky, 90, 012, 656049, Barnaul, Russian Federation \\ Tel.: 89831725918
}

On the basis of an eddy-current transformer of a transformer type, a measuring system is made, which makes it possible to estimate the possibility of using the vortex method for measuring the thickness of conductive and dielectric coatings placed on a conductive base. The design of the measuring system and measurement techniques is described. The article presents data demonstrating the dependence of the amplitude part of the signal on objects of different thicknesses; limiting object dimensions at which these measurements are expedient are experimentally established.

Keywords: thickness gauging, eddy-current converters, aluminum, copper, layered structures, electrical conductivity.

DOI: $10.17804 / 2410-9908.2017 .5 .063-069$

\section{References}

1. Neumeier P. Eddy current phase method for measuring the thickness of galvanic coatings. V mire nerazrushayushchego kontrolya, 2008, no. 2 (40), pp. 29-30. (In Russian).

2. Sjas'ko V.A., Bulatov A.S., Koroteev M.Ju., Solomenchuk P.V. Eddy Current Meter. RF Patent 2384839, 2011. (In Russian).

3. Bakunov A.S., Kaloshin V.A. Evolution of thickness coating measurement. Kontrol. Diagnostika, 2016, no. 1 (211), pp. 27-31. (In Russian). DOI: 10.14489/td.2016.01.pp.027-031

4. Bogdanov N.G., Prihod'ko V.A., Suzdal'cev A.I. Eddy current method of dual frequency control of products. Patent RF 2184931, 2002. (In Russian).

5. Malikov V.N., Dmitriev S.F., Sagalakov A.M., Ishkov A.V. Subminiature Eddy Current Transducers for Studying Metal-Dielectric Junctions. Instruments and experimental techniques, 2014, vol. 57, iss. 6, pp. 751-754. DOI: 10.1134/S0020441214060037

6. Dmitriev S.F., Katasonov A.O., Malikov V.N., Sagalakov A.M. Flaw Detection of Alloys Using the Eddy Current Method. Russian Journal of Nondestructive Testing, 2016, vol. 52, no. 1, pp. 32-37. DOI: 10.1134/S1061830916010058 
Подана в журнал: 28.06 .2017

УДК 620.179.14

DOI: $10.17804 / 2410-9908.2017 .5 .063-069$

\title{
СВЕРХМИНИАТЮРНЫЕ ВИХРЕТОКОВЫЕ ПРЕОБРАЗОВАТЕЛИ ДЛЯ ЗАДАЧ ТОЛЩИНОМЕТРИИ
}

\author{
С. Ф. Дмитриев ${ }^{1}$, А. В. Ишков ${ }^{2}$, А. О. Катасонов ${ }^{1}$, \\ В. Н. Маликов ${ }^{1 *}$, А. М. Сагалаков ${ }^{1}$ \\ ${ }^{I}$ ФББОУ ВО, Алтайский государственный университет, \\ Ленина, 61, Барнаул, Российская Федераџия \\ ${ }^{2}$ ФГБОУ ВО Алтайский государственный аграрный университет, \\ Красноармейский, 98, Барнаул, Российская Федерация \\ *Ответственный автор. Электронная почта: osys@ @e.com \\ Адрес для переписки: 656049, г. Барнаул, Красноармейский 90, 012; Российская Федерация \\ Тел.: 8-983-172-59-18
}

Новая измерительная система была изготовлена на базе вихретокового преобразователя. Система позволяет оценить возможность использования вихретокового метода для измерения толщины проводящих и диэлектрических покрытий, размещаемых на проводящей основе. Одним из важных преимуществ разработанной измерительной системы является возможность локального измерения толщин проводящих и непроводящих покрытий. В статье описаны подробно особенности проведения измерений, а также специфика измерительной системы. Также приведены данные, демонстрирующие зависимости амплитуды сигнала от объектов различной толщины и экспериментально установлены предельные размеры объектов, при которых данные измерения целесообразны.

Ключевые слова: толщинометрия, вихретоковые преобразователи, алюминий, медь, слоистые структуры, электрическая проводимость.

\section{1. Введение}

Для контроля толщины электропроводящих листов, пленок, пластин, покрытий на них, стенок труб, цилиндрических и сферических баллонов и определения величины воздушного зазора между пластинами из одного материала используются толщиномеры, основанные на методе вихревых токов.

Проведение вихретоковой толщинометрии объекта, являющегося по своей сути диэлектрическим или проводящим слоем (покрытием), размещенном на проводящем основании, требуется контроль, основанный на измерении фазы сигнала вихретокового преобразователя (ВТП). Использование данного метода, дает возможным определить толщину покрытия $d$. Как правило, при измерениях используется накладной ВТП. Такой преобразователь может содержать до трех обмоток: возбуждающая (создающая поле возбуждения), измерительная(предназначена непосредственно для проведения измерений) и компенсационная, предназначенная для снижения влияния возбуждающей обмотки на итоговый сигнал. При этом в качестве опорного сигнала становится возможным использовать электродвижущую силу в компенсационной обмотки преобразователя, а фаза отсчитывается исходя из синусоидального сигнала на возбуждающей обмотке. Такой подход используется с целью повышения точности измерения. Вносимая в измерительную обмотку электродвижущая сила имеет фазу, зависящую от геометрических параметров ВТП, используемой частоты тока $f$ на возбуждающей обмотке. В качестве важных параметров, оказывающих влияние на фазу, также являются зазор между преобразователем и покрытием, электрическая проводимость покрытия $\sigma_{1}$ и основания $\sigma_{1}$, а также магнитная проницаемость основания. 
К числу проблем такого подхода следует отнести вариацию величины электрической проводимости $\sigma_{l}$ в разных точках поверхности покрытия толщины $d$, а также при контроле различных объектов со схожими покрытиями. Это вызывает колебания величины фазы электродижущей силы, что приводит к увеличению погрешностей при измерении толщины покрытия. Различные виды отстроек (амплитудные, фазовые, амплитудно-фазовые) от влияний электрической проводимости $\sigma_{1} п р и$ этом практически не дают эффекта. Понижение частоты $f$ возбуждения тока на возбуждающей обмотки позволяет снизить влияние электрической проводимости. Однако в этом случае важным фактором влияния становится толщина подложки и магнитная проницаемость подложки.

Учитывая вышеприведенные факторы, необходимо выбрать такую частоту работы прибора, чтобы применить отстройку от колебаний электрической проводимости, не допуская при этом влияния магнитной проницаемости подложки. Оптимальным при этом становится измерять не фазу $\varphi$, а амплитуду $A$ сигнала, на которую параметры подложки оказывают существенно более низкое влияние, нежели на фазу.

При этом при измерении амплитуды сигнала ВТП с целью измерения толщины покрытия учитывается определенное значение электрической проводимости. Пример реализации такого способа контроля приведен в [4].

Цель данного исследования заключалась в оценке возможности локального $\left(2500\right.$ мкм²) $^{2}$ определения толщины проводящих и диэлектрических покрытий с использованием амплитуды сигналов ВТП.

\section{2. Описание установки}

Сверхминиатюрный ВТП $[5,6]$ разработан для локального исследования толщины различных покрытий в экспериментах, а также для выявления влияния различных покрытий на величину выходного сигнала.

Разработанный сверхминиатюрный ВТП состоит из сердечника, на который нанесены измерительные, возбуждающие и компенсационные обмотки. Обмотки и сердечник пропитываются компаундом, заключены они в шайбу из корунда. Это дает возможность увеличить стойкость преобразователя к механическому воздействию.

Для проведения тестирования разных проводящих материалов применяется разработанный преобразователь, который подключается к персональному компьютеру при помощи звуковой карты, используемой в роли генератора, и преобразователя сигнала. Сигнал при этом посылается непосредственно на возбуждающую обмотку.

Программное обеспечение способно управлять величиной сигнала, подаваемого на возбуждающую обмотку, и позволяет считывать значения напряжения с измерительной обмотки, которые с учетом калибровки переводятся в значения электропроводности. Разработанное программное обеспечение позволяет проводить измерение толщины проводящих и диэлектрических неферромагнитных покрытий и проводящих материалов

Намотанные витки ВТП состоят из медной проволоки, толщина которой равна 5 мкм. Сердечник изготовлен из феррита 2000 НМЗ со значением начальной магнитной проницаемости 2000 и имеет пирамидальную форму. Характеристики разработанного преобразователя позволяют добиться высокой локальности контроля, а именно - локализовать поле в пределах 2500 мкм². Разработанная система обеспечивает значительную глубину проникновения поля в исследуемый объект вплоть до величин 5 мм (при частотах порядка 500 Гц.)

Программное обеспечение, написанное на языке $\mathrm{C}++$ под операционную систему Windows, позволяет управлять сигналом на возбуждающей обмотке и принимать сигнал с измерительной обмотки. При помощи программного обеспечения возможно эффективно управлять сигналом, который подается непосредственно на возбуждающую обмотку. Также при помощи данного программного обеспечения можно принимать сигнал непосредственно с измерительной обмотки. Управлять подаваемым напряжением можно при помощи специ- 
ального микшера, встроенного в операционную систему Windows. При помощи микшера задаются параметры частоты и амплитуды синусоидального сигнала генератора. В свою очередь звуковая карта дает возможность расширить диапазон частот сигнала, который подается непосредственно на возбуждающую обмотку.

\section{3. Результаты эксперимента и их обсуждение}

Для тестирования новой измерительной системы осуществляли сканирование алюминиевого покрытия размещенного на основе, состоящей из меди. Измерения производились при частоте 700 Гц. При этом покрытие имело различную толщину, а толщина медного основания составляла 3 мм. На рис. 1 представлена зависимость величины сигнала от толщины алюминиевого покрытия на неферромагнитной основе. В случае увеличения значения толщины покрытия до значения в 1200 мкм размер сигнала уменьшается от 28 до 22 у.е., при значениях от 750 до 1500 мкм размер сигнала меньше сигнала монолита, это свидетельствует о том, что толщина покрытия недостаточно большая. При толщине диэлектрика от 1500 и до 2500 мкм размер сигнала постоянен и полностью соответствует показаниям величины поля от монолита.

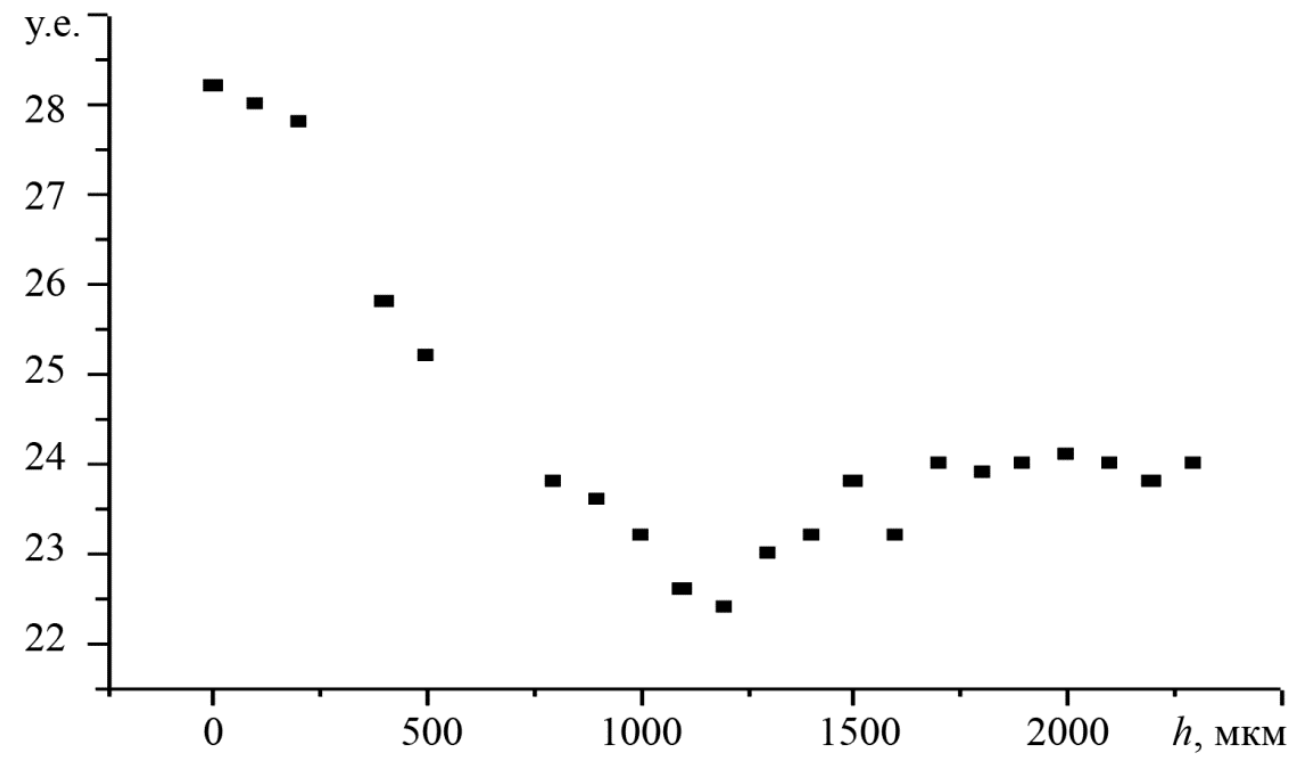

Рис. 1. Зависимость величины сигнала от толщины алюминиевого покрытия на медной основе

Также проводили эксперимент по определению зависимости толщины слоистого покрытия, в котором слои полиэтилена чередуются попеременно со слоями фольги. Объект исследования представлял чередование слоев алюминиевой фольги 20 мкм и полиэтилена 20 мкм. Измерения производились при частоте 1500 Гц.

На рис. 2. представлена зависимость интенсивности сигнала от толщины слоистого покрытия с чередованием слоев фольги и полиэтилена, размещаемых на медной основе. При изменении толщины слоистого покрытия от 0 до 100 мкм, величина поступающего от основы сигнала варьируется в пределах от 29 и до 24 у.е. В случае если толщина слоистого покрытия состоящего из полиэтилена и фольги варьируется от 100 до 250 мкм, вносимый сигнал демонстрирует более пологие значения. В диапазоне от 250 мкм до 400 мкм сигнал изменяется от 26 до 23 у.е., что обусловлено вкладом сигнала от слоистого покрытия и уменьшением вклада сигнала от медной основы. 


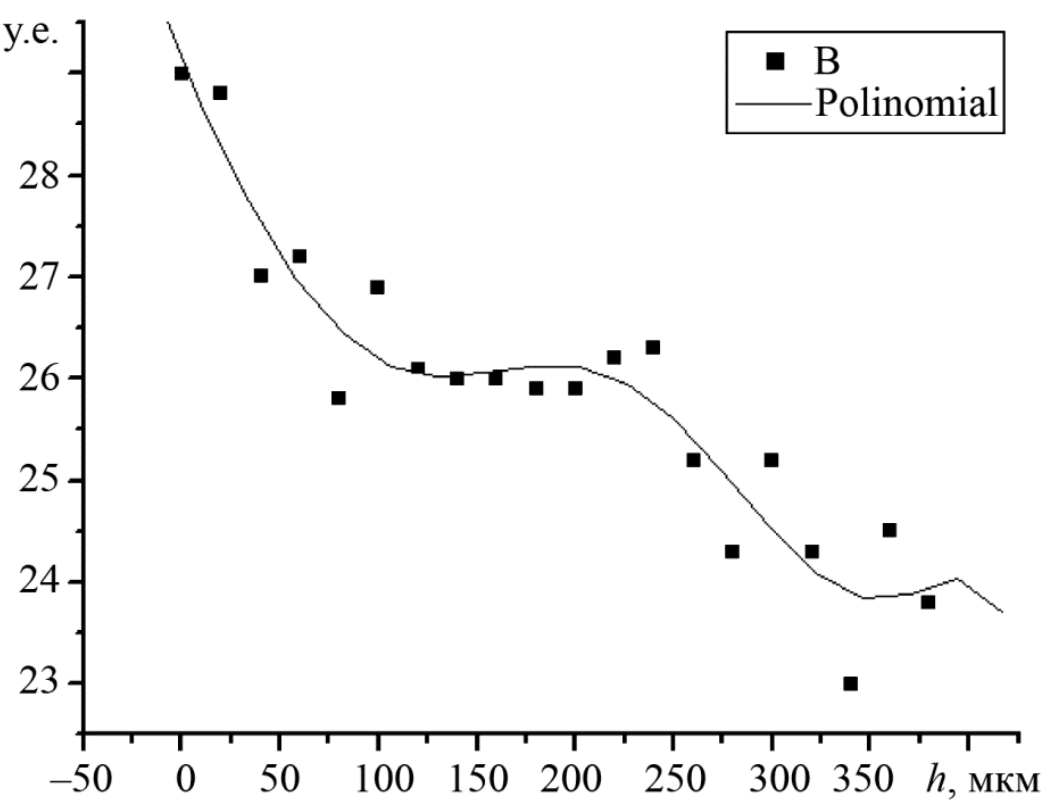

Рис. 2. Зависимость интенсивности сигнала при сканировании слоистой структуры

В третьем тестовом эксперименте осуществлялось сканирование образца, представляющего цельный объект из алюминия различной толщины. Измерения производились при частоте 500 Гц. На рис. 3 представлена зависимость величины сигнала от толщины образца из алюминия. С увеличением толщины образца увеличивается вклад в амплитуду сигнала более глубоких слоев образца. При изменении толщины от 100 до 1200 мкм величина сигнала возрастает от 7 до 25 у.е. При изменении толщины от 1200 до 2200 мкм величина сигнала постоянна и соответствует значению амплитуды от монолита (25,5 у.е.).

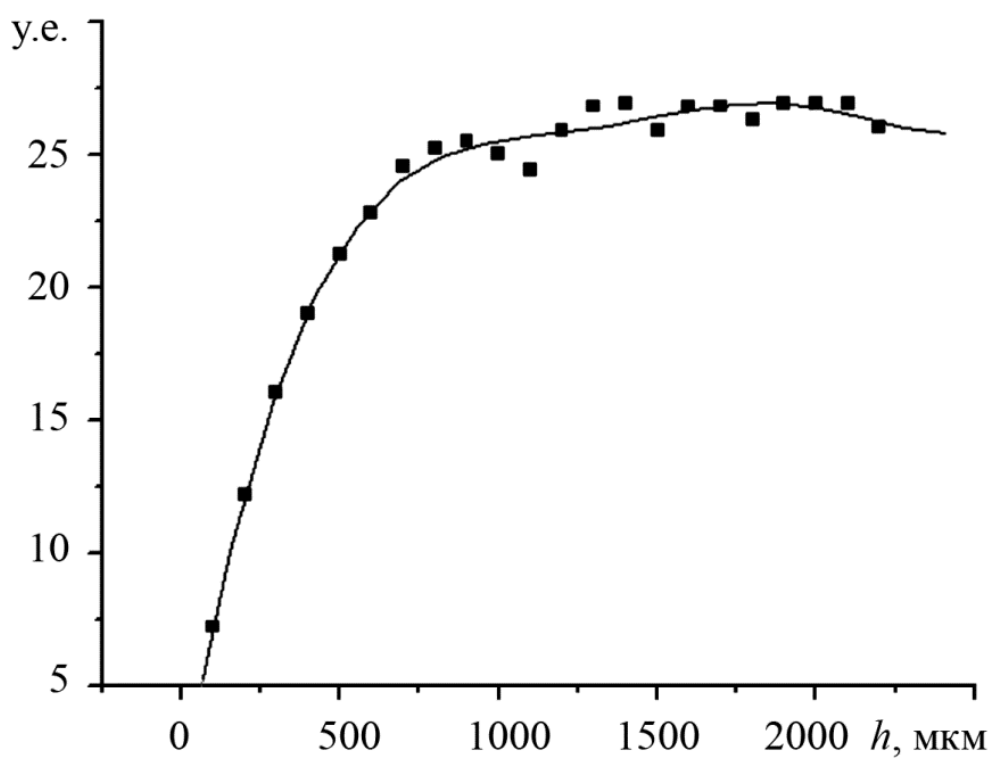

Рис. 3. Зависимость сигнала от толщины образца из алюминия

В заключительном исследовании эксперимент проводился также с медными образцами и лакокрасочным покрытием. На предварительно выпиленные образцы меди наносили слой лакокрасочного покрытия. Измерения производили при частоте 400 Гц. 


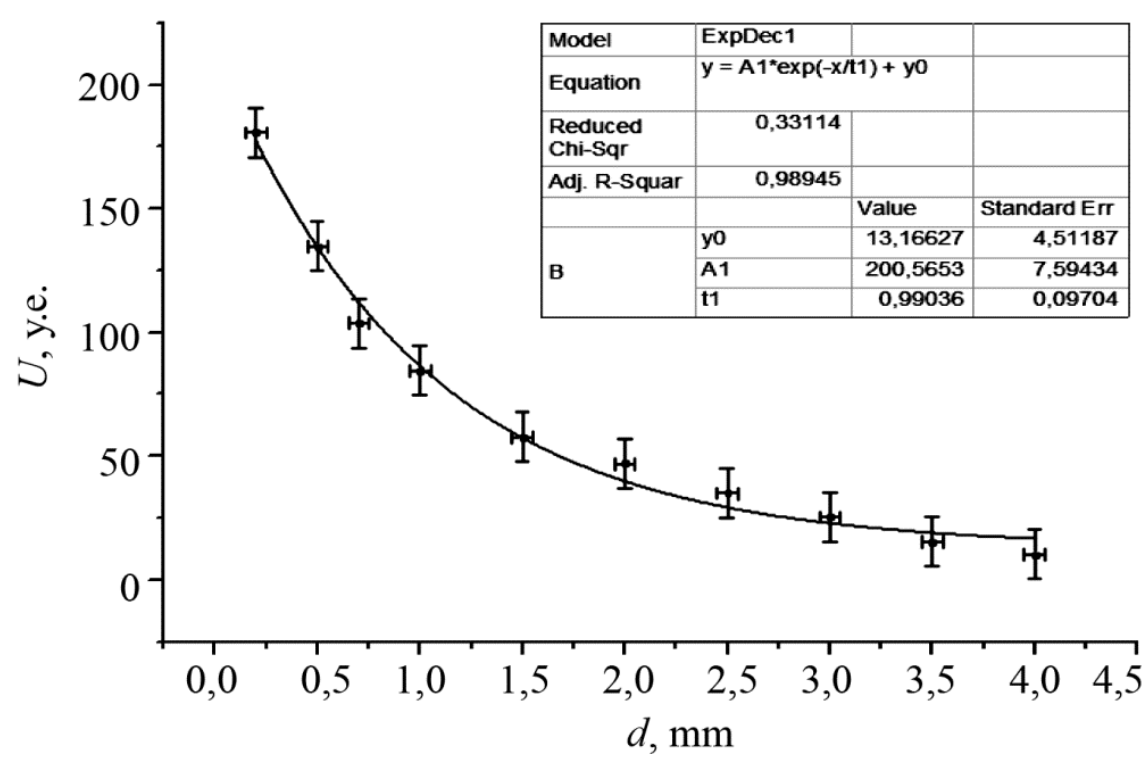

Рис. 4. Зависимость отклика вихретокового преобразователя от толщины диэлектрического покрытия (аппроксимированная экспоненциальной функцией)

Как видно из зависимости амплитуды сигнала от толщины диэлектрического покрытия (рис. 4), величина выходного сигнала быстро уменьшается при увеличении толщины покрытия. Эту зависимость можно аппроксимировать экспоненциальной функцией:

Графически данная зависимость и параметры аппроксимирующей функции представлены на рис. 4, из которого следует, что полученный сигнал уменьшается по экспоненте при возрастании толщины диэлектрического покрытия.

\section{4. Заключение}

Представленную измерительную систему использовали для исследования объектов, представляющих из себя проводящие и непроводящие покрытия, размещенные на проводящей основе, а также для измерения толщины монолитных проводящих объектов. Производилась оценка возможности локального определения толщины проводящих и диэлектрических покрытий с использованием амплитуды сигналов ВТП.

Установлено, что толщина покрытия оказывает влияние на сигнал вихретокового преобразователя. Это позволяет в перспективе использовать амплитудный метод контроля подобного класса объекта для локальных измерений толщины проводящих и непроводящих покрытий, а также других объектов.

\section{Благодарность}

Работа выполнена при поддержке Российского фонда фундаментальных исследований (код проекта - 17-48-220044) «Создание и исследование высокоэффективных композиционных и наноструктурированных упрочняющих покрытий»

\section{Литература}

1. Ноймайер П. Вихретоковый фазовый метод измерения толщины гальванических покрытий // В мире неразрушающего контроля. - 2008. - № 2 (40). - С. 29-30.

2. Вихретоковый измеритель: пат. 2384839 Рос. Федерация / Сясько В. А., Булатов А. С., Коротеев М. Ю., Соломенчук П. В., ЗАО «Константа». - № 2000548658/09 ; заявл. 13.10.08 ; опубл. 20.03.11, Бюл. № 8. -7 с. 
3. Бакунов А. С., Калошин В. А. Развитие вихретоковой толщинометрии защитных покрытий // Контроль. Диагностика. - 2016. - № 1 (211). - С. 27-31.

4. Вихретоковый способ двухчастотного контроля изделий: пат. 2184931 Рос. Федерация / Богданов Н. Г., Приходько В. А., Суздальцев А. И., Орловский государственный техн. унив. № 2000625485/09 ; заявл. 03.02.00 ; опубл. 10.07.02, Бюл. № 19. - 5 с.

5. Subminiature Eddy Current Transducers for Studying Metal-Dielectric Junctions / V. N. Malikov, S. F. Dmitriev, A. M. Sagalakov, A. V. Ishkov // Instruments and Experimental Techniques. - 2014. - Vol. 57, iss. 6. - P. 751-754. - DOI: 10.1134/S0020441214060037

6. Flaw Detection of Alloys Using the Eddy Current Method / S. F. Dmitriev, A. O. Katasonov, V. N. Malikov, A. M. Sagalakov // Russian Journal of Nondestructive Testing. 2016. - Vol. 52, no. 1. - P. 32-37. - DOI: 10.1134/S1061830916010058 